\section{Les cardiomyocytes adultes se réveillent}

\section{Un grand pas vers la régénération cardiaque}

Céline Mias, Céline Galés
Institut des maladies métaboliques et cardiovasculaires, Inserm/UPS UMR 1048, Université Toulouse III Paul Sabatier, bâtiment L3, 1, avenue Jean Poulhès, BP 84225 , 31432 Toulouse Cedex 4, France. celine.mias@inserm.fr celine.gales@inserm.fr

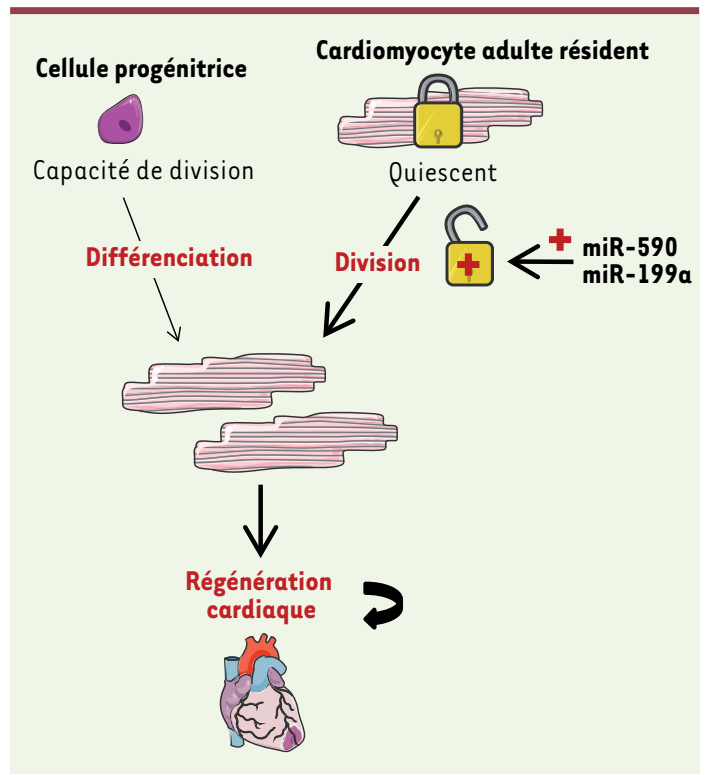

Figure 1. Les deux voies cellulaires de la régénération cardiaque. phie génétique, a permis de suivre simultanément le renouvellement et l'origine des cardiomyocytes adultes dans des conditions naturelles d'homéostasie ou en situation pathologique. En effet, suite à l'administration in vivo de $\left[{ }^{15} \mathrm{~N}\right]$-thymidine par pulse-chase, la technique de MIMS [8], en générant des images tissulaires quantitatives de très haute résolution subcellulaire, a permis de quantifier très finement le taux de réplication des cardiomyocytes présents dans le tissu cardiaque par l'identification précise de leurs noyaux ayant incorporé l'isotope. La stratégie de cartographie génétique, quant à elle, utilise un modèle de souris double transgéniques (MerCreMer-Z\&G) ${ }^{1}$ $[9,10]$ qui permet de marquer les car-

${ }^{1}$ B6129-Tg (myh6-Cre/Esrl)1 Jmk/J (= MerCreMer) x B6.Cg$\mathrm{Tg}($ ACTB-Bgeo/GFP)21Lbe/J (= ZEG). diomyocytes adultes de façon spécifique et irréversible en induisant l'expression de la protéine fluorescente verte GFP après un traitement par le tamoxifène. En utilisant ces souris, les auteurs ont ainsi pu discriminer précisément l'origine des cardiomyocytes néoformés, dérivant soit de cardiomyocytes adultes différenciés $\left(\mathrm{GFP}^{+}\right)$, soit de la différenciation d'un pool de progéniteurs $\left(\mathrm{GFP}^{-}\right)$. Les résultats de l'étude combinant les deux techniques sont très clairs et montrent ainsi que seule une majorité de cardiomyocytes préexistants sont capables d'initier spontanément un programme de division intrinsèque et se renouvellent dans des cœurs de souris jeunes, même si le taux de renouvellement reste faible $(0,76 \%$ par an) dans des conditions normales d'homéostasie et diminue avec l'âge. Ce 
résultat confirme une étude antérieure menée chez l'homme grâce à une technique similaire de marquage isotopique radioactif au ${ }^{14} \mathrm{C}[11]$. Dans une situation d'infarctus du myocarde, le taux de renouvellement des cardiomyocytes augmente mais n'atteint cependant que $3 \%$ par an. De façon intéressante, dans ce cas précis, la majorité des cardiomyocytes résidents qui rompent leur état de quiescence et entrent à nouveau dans le cycle cellulaire en réponse aux dommages cardiaques ischémiques ne réalisent pas un cycle de division complet : ils se répliquent mais ne sont pas capables de finaliser l'étape terminale de cytokinèse, c'est-à-dire l'individualisation en deux cellules filles. Ces résultats confirment donc définitivement que le cœur possède un pouvoir naturel de régénération reposant essentiellement sur le renouvellement de ses cardiomyocytes différenciés préexistants, bien que ce processus reste trop limité et insuffisant pour réparer un tissu cardiaque endommagé. Cette étude redonne ainsi de l'élan aux stratégies d'utilisation des cardiomyocytes résidents pour stimuler la régénération cardiaque avec notamment l'espoir, dans le futur, de développer des outils permettant d'amplifier ce phénomène.

- La seconde étude [7], publiée par l'équipe de Mauro Giacca, répond directement à la question de la modulation du taux de renouvellement des cardiomyocytes. Elle applique une stratégie basée sur l'utilisation de microARN permettant de rompre l'état de quiescence postmitotique des cardiomyocytes adultes résidents et de stimuler leur division. À partir d'un crible aléatoire à grande échelle de 875 microARN, les auteurs en ont identifié 204 qui augmentent significativement la prolifération des cardiomyocytes néonataux de rats. Parmi ces 204 microARN, seuls 40 fonctionnent également chez la souris nouveau-né en favorisant l'incorporation d' $\varepsilon d U$ (un analogue de la thymidine qui s'incorpore dans l'ADN en cours de réplication) et le marquage $\mathrm{Ki}-67$ (un marqueur de la prolifération) dans les cardiomyocytes. Les auteurs ont finalement sélectionné deux de ces microARN (hsa-miR-590-3p et hsa-miR-199a-3p) qui stimulent considérablement - de 13 à $20 \%$ - l'incorporation d'EdU dans les cardiomyocytes de souris âgées de sept jours, âge auquel la prolifération des cardiomyocytes est généralement quasi-inexistante. La réintroduction de ces microARN, naturellement faiblement exprimés dans les cardiomyocytes adultes, réoriente de façon drastique les cardiomyocytes matures postnataux dans le cycle cellulaire et induit leur prolifération. Après un infarctus du myocarde, l'injection in vivo de ces microARN dans la zone péri-infarctus favorise de façon spectaculaire la régénération du tissu cardiaque ischémié : la taille de la zone nécrosée est réduite de $50 \%$ et la fonction cardiaque s'améliore de façon significative, résultats en parfait accord avec les effets promitogènes de ces microARN sur les cardiomyocytes adultes. L'étude montre également que les effets bénéfiques de ces deux microARN dépendent de multiples cibles cellulaires qui semblent coopérer pour maintenir l'état de quiescence des cardiomyocytes. En effet, bien que l'analyse des transcrits modulés par ces microARN révèle une augmentation des gènes régulant le cycle cellulaire, la prolifération cellulaire et la réplication de I'ADN, l'invalidation individuelle de chacun de ces gènes ne permet pas de reproduire les effets mitogènes des deux microARN sur les cardiomyocytes. Les auteurs démontrent également que les effets promitogènes des deux microARN sont spécifiques aux cardiomyocytes puisqu'ils n'affectent pas d'autres types cellulaires cardiaques tels que les fibroblastes. À ce jour, aucune étude de médecine régénérative cardiaque n'a obtenu un tel succès thérapeutique chez les mammifères. Bien que les auteurs n'aient pu parvenir à identifier la cible protéique des microARN dans cette étude, ils montrent qu'il est possible de rompre la quiescence des cardiomyocytes. Ces résultats ouvrent donc un champ d'investigation très prometteur pour une future application chez l'homme. La recherche concernant la reprogrammation du cycle cellulaire des cardiomyocytes résidents prend désormais une importance considérable car elle pourrait pallier les problèmes classiquement rencontrés aujourd'hui dans les protocoles de thérapie cellulaire qui utilisent des cellules exogènes, et notamment la différenciation non contrôlée et inadaptée de ces cellules dans le cœur à long terme ainsi que les problèmes d'ordre éthique.

Le cœur de mammifère régénère... Cependant, la caractérisation précise des mécanismes moléculaires contrôlant l'état postmitotique des cardiomyocytes et de leurs modulateurs constitue maintenant un enjeu crucial pour le futur développement d'outils thérapeutiques en médecine régénérative cardiaque. $\diamond$

Adult cardiomyocytes wake up:

a big step towards cardiac

regeneration

\section{LIENS D'INTÉRÊT}

Les auteurs déclarent n'avoir aucun lien d'intérêt concernant les données publiées dans cet article.

\section{RÉFÉRENCES}

1. Laflamme MA, Murry CE. Heart regeneration. Nature $2011 ; 473: 326-35$.

2. Mias C, Genet G, Oathak A, et al. Le réveil des cardiomyocytes adultes résidents : une nouvelle piste de régénération cardiaque? Med Sci (Paris) 2012 ; 28 : 1103-9.

3. Bersell K, Arab S, Haring B, Kuhn B. Neuregulinl/ErbB4 signaling induces cardiomyocyte proliferation and repair of heart injury. Cell $2009 ; 138: 257-70$.

4. Kuhn B, del Monte F, Hajjar RJ, et al. Periostin induces proliferation of differentiated cardiomyocytes and promotes cardiac repair. Nat Med $2007 ; 13$ : 962-9.

5. Engel FB, Hsieh PC, Lee RT, Keating MT. FGFl/p38 MAP kinase inhibitor therapy induces cardiomyocyte mitosis, reduces scarring, and rescues function after myocardial infarction. Proc Natl Acad Sci USA 2006 ; 103: 15546-51.

6. Senyo SE, Steinhauser ML, Pizzimenti CL, et al. Mammalian heart renewal by pre-existing cardiomyocytes. Nature $2013 ; 493$ : 433-6.

7. Eulalio A, Mano M, Ferro MD, et al. Functional screening identifies miRNAs inducing cardiac regeneration. Nature $2012 ; 492: 376-81$.

8. Lechene C, Hillion F, McMahon G, et al. High-resolution quantitative imaging of mammalian and bacterial cells using stable isotope mass spectrometry. J Biol $2006 ; 5: 20$. 


\section{RÉFÉRENCES}

9. Hsieh PC, Segers VF, Davis ME, et al. Evidence from a genetic fate-mapping study that stem cells refresh adult mammalian cardiomyocytes after injury. Nat Med $2007 ; 13: 970-4$.
10. Loffredo FS, Steinhauser ML, Gannon J, Lee RT. Bone marrow-derived cell therapy stimulates endogenous cardiomyocyte progenitors and promotes cardiac repair. Cell Stem Cell $2011 ; 8$ : 389-98.
11. Bergmann 0, Bhardwaj RD, Bernard S, et al. Evidence for cardiomyocyte renewal in humans. Science 2009 ; $324: 98-102$

\section{NOUVELLE}

Contribution au développement
de nouvelles thérapies
contre le cancer colorectal
L'initiative CReMcc
Alain Bruno

$>$ En dépit d'avancées certaines dans la compréhension des mécanismes moléculaires qui sous-tendent l'apparition et la propagation du cancer colorectal, et de l'émergence de thérapies ciblées, le traitement de cette pathologie demeure encore insatisfaisant. Ceci est particulièrement vrai pour les cancers colorectaux localement évolués ou compliqués d'emblée de métastases à distance. Plusieurs paramètres peuvent être incriminés : (1) les variations interindividuelles de pharmacocinétique des drogues anticancéreuses, (2) la diversité des cancers colorectaux, en rapport notamment avec leur profil moléculaire, et (3) I'hétérogénéité intratumorale [10] ainsi que le temps de doublement intrinsèque des tumeurs. C'est avec l'objectif ambitieux de contribuer à l'émergence de nouvelles thérapies du cancer colorectal qu'est née l'initiative CReMEC (centre de ressource de modèles expérimentaux de cancer).

\section{Des partenaires unis}

\section{contre le cancer colorectal}

Le CReMEC est l'un des premiers projets labellisés par le pôle de compétitivité Medicen Paris Région en 2005. II associe des acteurs industriels (Ipsen, Sanofi, Servier, Oncodesign) et dix équipes académiques du Cancéropôle Île-de-
France issues de I'AP-HP, du CNRS, de I'Inserm, de l'Institut Curie, de I'Institut Gustave Roussy et de l'université Paris VII Diderot.

L'enjeu était de développer chez la souris une collection de tumeurs coliques humaines représentant la diversité du panel rencontré en pratique clinique. Ce projet a bénéficié d'un financement de 2,4 M€ de la part de l'État français dans le cadre d'un appel FUI (fonds uniques interministériels) et d'un investissement industriel de 2,5 M€. Sa réussite doit beaucoup à la synergie entre les différents partenaires qui ont mutualisé leurs compétences à chacune des étapes, depuis l'exérèse de la tumeur chez le patient jusqu'à la caractérisation moléculaire et pharmacologique des modèles établis chez la souris. Le projet a permis de créer une collection de 54 modèles primaires de cancers colorectaux, ainsi qu'une biobanque de 5000 échantillons. L'ensemble de l'information tirée de la caractérisation histoclinique, moléculaire et pharmacologique des modèles a été consigné dans une base de données informatique.

\section{Caractéristiques cliniques des patients} et établissement des tumeurs chez la souris immunodéprimée Au total, 85 échantillons de cancers colorectaux ont été prélevés au bloc opératoire (échantillons issus de tumeurs primaires, de carcinoses péritonéales ou de métastases à distance) et ont été immédiatement greffés par voie souscutanée sur le flanc de souris nude. La collection de xénogreffes dérivées des tumeurs de patients reflétait sensiblement la diversité des cas cliniques de cancers colorectaux en termes de genre, de sexe, de dosage de l'antigène carcino-embryonnaire avant la chirurgie d'exérèse, de localisation de la tumeur primaire dans le tractus intestinal, d'envahissement des ganglions lymphatiques et de présence de métastases synchrones. Sur les 85 tumeurs, 54 ont été greffées avec succès $(63,5 \%)$. Aucun impact du temps d'ischémie froide (inférieur à 24 heures) n'a été observél. Une biobanque a été établie pour tous les modèles de xénogreffes avec un taux de succès à la décongélation (capacité des fragments tumoraux à subir un cycle de congélation-décongélation et à se développer à nouveau après greffe par voie sous-cutanée) de $92,3 \%$, proche du taux observé pour les tumeurs directement issues de patients. Dans cette collection, on compte cinq couples tumeur primaire/métastase hépatique synchrone,

\footnotetext{
1 L'ischémie froide est le laps de temps pendant lequel le greffon est placé en hypothermie pour diminuer les conséquences de l'absence de perfusion de l'organe.
}

en oncologie, Institut de recherches Servier, 125, chemin de Ronde,

78290 Croissy-sur-Seine, France. alain.bruno@fr.netgrs.com 